Pacific Journal of Mathematics

EACH COMPACT ORIENTABLE SURFACE OF POSITIVE
GENUS ADMITS AN EXPANSIVE HOMEOMORPHISM 


\section{EACH COMPACT ORIENTABLE SURFACE OF POSITIVE GENUS ADMITS AN EXPANSIVE HOMEOMORPHISM}

\section{Thomas O'Brien and William Reddy}

It is known that the torus and the orientable surface of genus 2 admit expansive homeomorphisms. In this paper it is shown that all compact orientable surfaces of positive genus admit such homeomorphisms. It remains unknown whether $S^{2}$ admits such a map. By taking products expansive homeomorphisms on higher dimensional manifolds are exhibited. Finally dynamical properties of these examples are discussed. Among these are occurrence and nature of periodic points, topological entropy and existence of interesting minimal sets.

A homeomorphism $f$ of a compact metric $(d)$ space $X$ onto itself will be called expansive with expansive constant $c>0$ (or just expansive) provided that for each pair of distinct points $x, y$ in $X$ there is an integer $n$ such that $d\left[f^{n}(x), f^{n}(y)\right]>c$. We denote by $M_{k}$ the compact orientable surface of genus $k$. In [8] Reddy exhibited an expansive homeomorphism, $f$, on the torus, $M_{1}$. This mapping is that induced on the torus by the linear mapping of the plane whose matrix is:

$$
\left(\begin{array}{ll}
1 & 1 \\
1 & 2
\end{array}\right)
$$

In [6] O'Brien exhibited an expansive homeomorphism, $g$, on $M_{2}$. This map was obtained by lifting $f^{3}$ through a branched covering mapping, $\phi$, from $M_{2}$ onto the torus. If we consider these spaces as spheres with handles imbedded in $R^{3}$ then in each horizontal plane $\phi$ is the mapping which sends $z$ into $z^{2}$ ( $z$ a complex number). Thus the expansive homeomorphism $g$ is a lift of the expansive homeomorphism on $M_{1}$ induced by the matrix.

$$
A=\left(\begin{array}{ll}
1 & 1 \\
1 & 2
\end{array}\right)^{3}=\left(\begin{array}{rr}
5 & 8 \\
8 & 13
\end{array}\right) .
$$

The triple iterate of the torus homeomorphism has a fixed point, $m$, which is not a branch point image. In [6] this point is chosen as a base point. The lift to $M_{2}$ can be chosen to leave $n \in \phi^{-1}(m)$ fixed. We will use $n$ as base point for the fundamental group of $M_{2}$ in $\S 2$.

2. The examples. In this section we prove the existence of expansive homeomorphisms on many compact manifolds. The technique 
is to exhibit, for any $n>2$, an expansive homeomorphism of $M_{2}$ (which will be an iterate of the above-mentioned map on $M_{2}$ ) which lifts through a covering map to $M_{k}$. According to [5, Corollary 3.5] the lift will be expansive. Then since the product of expansive homeomorphisms is expansive, it will follow that any product of orientable surfaces of positive genus admits an expansive homeomorphism.

THEOREM 2.1. Each compact orientable surface of positive genus admits an expansive homeomorphism.

Proof. Let $f, g$ and $\phi$ be the maps mentioned in the preliminaries. Choose generators $\alpha, \beta$ for $\pi_{1}\left(M_{1}, m\right)$ where $\alpha$ is covered by the segment from $(0,0)$ to $(1,0)$ in the plane and $\beta$ by the segment from $(0,0)$ to $(0,1)$. This represents $\pi_{1}\left(M_{1}, m\right)$ as $Z \oplus Z$ (where $Z$ denotes the integers) in such a way that $f_{*}: \pi_{1}\left(M_{1}, m\right) \rightarrow \pi_{1}\left(M_{1}, m\right)$ is given by the matrix

$$
\left(\begin{array}{ll}
1 & 1 \\
1 & 2
\end{array}\right)
$$

An easy induction shows that, for each positive integer $j, f_{*}^{3 j}$ is given by the matrix

$$
\left(\begin{array}{ll}
f_{6 j-1} & f_{6 j} \\
f_{6 j} & f_{6 j+1}
\end{array}\right)
$$

where $f_{i}$ denotes the $i^{\text {th }}$ Fibonacci number $\left(f_{1}=1, f_{2}=1, f_{i+1}=f_{i}+f_{i-1}\right)$. Now choose generators $\alpha_{1}, \alpha_{2}, \beta_{1}, \beta_{2}$ for $\pi_{1}\left(M_{2}, n\right)$ such that $\phi_{*}\left(\alpha_{i}\right)=\alpha$ and $\phi_{*}\left(\beta_{i}\right)=\beta$. Define a homomorphism $P: \pi_{1}\left(M_{2}, n\right) \rightarrow Z \oplus Z$ by $P(\gamma)=$ $(a, b)$ where $a$ is the sum of the exponents of $\alpha_{1}$ and $\alpha_{2}$ and $b$ is the sum of the exponents of $\beta_{1}$ and $\beta_{2}$ in a word representing $\gamma$. Because of the defining relation for the group $\pi_{1}\left(M_{2}, n\right), P$ is independent of the word representing $\gamma$. Now if $P(\gamma)=(a, b)$ then $\phi_{*}(\gamma)=\alpha^{a} \beta^{b}$. Also

$$
f_{*}^{3 j}\left(\alpha^{a} \beta^{b}\right)=\left(\alpha^{f_{6 j-1}{ }^{a+f_{6 j} b}} \beta^{f_{6 j} j^{a+f_{6 j+1} b}}\right) .
$$

Thus by commutativity $\left(f^{3 j} \phi=\phi g^{j}\right)$ and the fact that kernel $P=$ kernel $\phi_{*}$ it follows that $P\left(g_{*}^{j}(\gamma)\right)=\left(f_{6 j-1} a+f_{6 j} b, f_{6 j} a+f_{6 j+1} b\right)$.

Let a surface $M_{k+1}(k \geqq 2)$ be given. Consider the normal subgroup $G_{k}$ of $\pi_{1}\left(M_{2}, n\right)$ given by

$$
G_{k}=\left\{\gamma \in \pi_{1}\left(M_{2}, n\right): P(\gamma)=(k a, b)\right\} .
$$

The index of $G_{k}$ is $k$. Thus $g_{*}^{j}\left(G_{k}\right)$ is also a subgroup of index $k$ in $\pi_{1}\left(M_{2}, n\right)$. We now determine conditions on $k$ such that $g_{*}^{j}\left(G_{k}\right)=G_{k}$. When this happens, $g^{j}$ lifts to the covering space of $M_{2}$ associated 
with the subgroup $G_{k}$. Through consideration of the Euler characteristic, we infer that this space is $M_{k+1}$.

If $P(\gamma)=(k a, b)$ then $P\left(g_{*}^{j} \gamma\right)=\left(f_{6 j-1} k a+f_{6 j} b, f_{6 j} k a+f_{6 j+1} b\right)$. Thus a necessary and sufficient condition for $g_{*}^{j}(\gamma)$ to be in $G_{k}$ is that $k$ divide $f_{6 j}$. Therefore, for existence of a lifting of an expansive homeomorphism on $M_{2}$ to the surface of genus $k+1$ it is sufficient that $k$ divide $f_{6 j}$ for some $j$. According to [9], for any $k$, the Fibonacci sequence $\bmod k$ is periodic and if $j$ is the period then $f_{j} \equiv 0(\bmod k)$. Thus, by the periodicity, $f_{6 j} \equiv 0(\bmod k)$. Therefore $k$ does divide some $6 j^{\text {th }}$ Fibonacci number. It follows that $g^{j}$ lifts to a homeomorphism on $M_{k+1}$. This homeomorphism is expansive by [5, Corollary 3.5].

COROLlaRY 2.2. Let $M$ be a topological product whose factors are compact orientable surfaces of positive genus. Then $M$ admits an expansive homeomorphism.

3. Properties. In this section we prove several propositions concerning dynamical properties of the examples just constructed.

Proposition 3.1. In all of the examples the set of periodic points is dense.

Proof. For the torus case this is well known. See [7, p. 758]. Any lift to $M_{n}$ through a pseudo-covering map must have dense periodic points since the fibre over a periodic point will consist of periodic points. For the higher dimensional manifolds the set of periodic points is just the product of the periodic sets in the factors and is therefore dense.

DEFINITION 3.2. A fixed point, $x$, of an expansive homeomorphism, $\phi$, is called a saddle point if there exist $p \neq x$ and $q \neq x$ such that $p$ is positively assymptotic to $x$ and $q$ is negatively assymptotic to $x$. If $x$ is a periodic point with period $m$ and $x$ is a saddle point of $f^{m}$ we will say that $x$ is of saddle type.

Proposition 3.3. For the examples in $\S 2$, all periodic points are of saddle type.

Proof. According to Theorem 9 in [3] all periodic points will be of saddle type if the homeomorphism preserves a continuous Borel probability measure which is positive on open sets. Since an automorphism of an abelian group space preserves Haar measure we have our result in the toral case. We can use the pseudo-covering mapp- 
ings to lift the measure on the torus to the higher genus spaces so that the lifted expansive homeomorphism preserves the measure. Thus all periodic points on the $M_{k}$ are of saddle type. Clearly when we take products all periodic points will be of saddle type.

Next we show that all of our examples have nonzero topological entropy. See [1] for definitions and results about topological entropy. According to K. R. Berg [2] the entropy of our toral maps is not 0 . We wish to show that all of our examples have non-zero topological entropy. The following is a special case of Theorem 5 in [1].

THEOREM 3.5. Suppose $f: M \rightarrow M$ and $g: N \rightarrow N$ are continuous, $M$ and $N$ are compact and $\phi: M \rightarrow N$ is open and onto and that $g \phi=\phi f$. Ther $h(g) \leqq h(f)$.

Proposition 3.6. The examples of $\S 2$ all have nonzero topological entropy.

Proof. It follows from the construction in Theorem 2.1., Theorem 3.5. and Berg's result that this conclusion holds for the homeomorphisms constructed on each of the surfaces $M_{k}$. Since entropy satisfies the relation $h(f \times g)=h(f)+h(g)$ ([1]), the proposition is valid.

Finally we consider minimal sets. All of our examples have nonperiodic minimal sets. For each space $M$ we exhibit an expansive homeomorphism (an iterate of one of those given $\S 2$ ) such that some subspace restriction is a Sturmiam minimal set [4; pp. 111-113].

Proposition 3.7. Each space considered in $\$ 2$ admits an expansive homeomorphism $f$ with nonperiodic minimal sets.

Proof. Each of our examples $f$ on $M_{k}$ projects through a pseudocovering mapping, $\phi$, onto a torus automorphism, $g$. According to [7, Th. 5.5] there is a Cantor set $\Lambda \subset M_{1}$ and an integer $m$ such that $g^{m}(\Lambda)=\Lambda$ and $g^{m}$ restricted to $\Lambda$ is topologically a shift automorphism. There is a subset $L$ of $A$ which is totally minimal with respect to $g^{m}[4,12.63] . \quad L$ is compact and contains no fixed points. In particular $\phi\left(B_{\phi}\right) \cap L$ is empty. Thus $L$ is contained in a simply connected subset $U$ of $M_{1}-\phi\left(B_{\phi}\right)$. Each arc component of $\phi^{-1}(U)$ in $M_{k}-B_{\phi}$ maps homeomorphically onto $U$. There arc $2 k-2$ such arc components. Thus there are $2 k-2$ copies, $L_{i}$, of $L$ in $M_{k}$. The set $\bigcup_{i=1}^{2 k-2} L_{i}$ is invariant under $f$. We can cover $L$ by a finite collection of disjoint elementary neighborhoods $U_{j}$. These lift to open sets 
$V_{i j}$ such that $L_{i} \subset_{j}^{U} V_{i j}$. The effect of $f$ on the collection $\left\{V_{i j}\right\}$ is, essentially, to permute these sets. Thus for some iterate $f^{n}$ of $f$ there is an invariant copy of $L$ and $f^{n}$ is topologically an iterate of the shift automorphism. Since the Sturmian minimal sets are totally minimal we have exhibited nonperiodic minimal sets for each $M_{k}$. For a product $M \times N$ we can choose a subset $L \times\left\{x_{0}\right\}$ where $L$ is a minimal orbit closure of $M$ and $x_{0}$ is a fixed point. Thus all of our examples contain nonperiodic minimal sets.

\section{REFERENCES}

1. R. L. Adler, A. G. Konheim, M. H. McAndrew, Topological entropy, Trans. Amer. Math. Soc. 114 (1965), 309-319.

2. K. R. Berg, Entropy of torus automorphisms, Topological Dynamics, An International Symposium, Ed. J. Auslander and W. Gottschalk, W. A. Benjamin (1968).

3. B. F. Bryant, P. Walters, Asymptotic properties of expansive homeomorphisms, Math. Systems Theory 3 (1969), 60-66.

4. W. H. Gottschalk and G. A. Hedlund, Topological Dynamics, Amer. Math. Soc. Colloq. Publ., vol. 36, Amer. Math. Soc., Providence, R. I., 1955.

5. E. Hemmingsen and W.L. Reddy, Lifting and projecting expansive homeomorphisms, Math. Systems Theory 2 (1968), 7-15.

6. T. O'Brien, Expansive homeomorphisms on compact manifolds. Proc. Amer. Math. Soc. 24 (1970), 767-771.

7. S. Smale, Differentiable dynamical systems, Bull. Amer. Math. Soc. 73 (1967), 747-817.

8. W. Reddy, The existence of expansive homeomorphisms on manifolds, Duke Math. J. 32 (1965), 627-632.

9. D. D. Wall, Fibonacci series modulo $m$, Amer. Math. Monthly 67 (1960), 525-532.

Received March 20, 1970. The second author was supported in part by Army Research Office-Durham Grant 31-124-G892, S2, and NSF Grant GP-23105.

Bowling Green State University

InStitute FOR ADVANCED StUdy aNd WeSLEyAN University 



\section{PACIFIC JOURNAL OF MATHEMATICS}

\section{EDITORS}

\author{
H. SAMELSON \\ Stanford University \\ Stanford, California 94305 \\ Richard Pierce \\ University of Washington \\ Seattle, Washington 98105
}

\author{
J. DugundJI \\ Department of Mathematics \\ University of Southern California \\ Los Angeles, California 90007 \\ RICHARD ARENS \\ University of California \\ Los Angeles, California 9.0024
}

\section{ASSOCIATE EDITORS}

\begin{tabular}{|c|c|}
\hline E. F. BECKENBACH & K. YoshidA \\
\hline \multicolumn{2}{|c|}{ SUPPORTING INSTITUTIONS } \\
\hline UNIVERSITY OF BRITISH COLUMBIA & STANFORD UNIVERSITY \\
\hline CALIFORNIA INSTITUTE OF TECHNOLOGY & UNIVERSITY OF TOKYO \\
\hline UNIVERSITY OF CALIFORNIA & UNIVERSITY OF UTAH \\
\hline MONTANA STATE UNIVERSITY & WASHINGTON STATE UNIVERSITY \\
\hline UNIVERSITY OF NEVADA & UNIVERSITY OF WASHINGTON \\
\hline NEW MEXICO STATE UNIVERSITY & $* \quad * \quad *$ \\
\hline OREGON STATE UNIVERSITY & AMERICAN MATHEMATICAL SOCIETY \\
\hline UNIVERSITY OF OREGON & CHEVRON RESEARCH CORPORATION \\
\hline OSAKA UNIVERSITY & TRW SYSTEMS \\
\hline UNIVERSITY OF SOUTHERN CALIFORNIA & NAVAL WEAPONS CENTER \\
\hline
\end{tabular}

The Supporting Institutions listed above contribute to the cost of publication of this Journal, but they are not owners or publishers and have no responsibility for its content or policies.

Mathematical papers intended for publication in the Pacific Journal of Mathematics should be in typed form or offset-reproduced, (not dittoed), double spaced with large margins. Underline Greek letters in red, German in green, and script in blue. The first paragraph or two must be capable of being used separately as a synopsis of the entire paper. The editorial "we" must not be used in the synopsis, and items of the bibliography should not be cited there unless absolutely necessary, in which case they must be identified by author and Journal, rather than by item number. Manuscripts, in duplicate if possible, may be sent to any one of the four editors. Please classify according to the scheme of Math. Rev. Index to Vol. 39. All other communications to the editors should be addressed to the managing editor, Richard Arens, University of California, Los Angeles, California, 90024.

50 reprints are provided free for each article; additional copies may be obtained at cost in multiples of 50 .

The Pacific Journal of Mathematics is published monthly. Effective with Volume 16 the price per volume (3 numbers) is $\$ 8.00$; single issues, $\$ 3.00$. Special price for current issues to individual faculty members of supporting institutions and to individual members of the American Mathematical Society: $\$ 4.00$ per volume; single issues $\$ 1.50$. Back numbers are available.

Subscriptions, orders for back numbers, and changes of address should be sent to Pacific Journal of Mathematics, 103 Highland Boulevard, Berkeley, California, 94708.

PUBLISHED BY PACIFIC JOURNAL OF MATHEMATICS, A NON-PROFIT CORPORATION

Printed at Kokusai Bunken Insatsusha (Internatıonal Academic Printing Co., Ltd.), 7-17, Fujimi 2-chome, Chiyoda-ku, Tokyo, Japan. 


\section{Pacific Journal of Mathematics}

\section{Vol. 35, No. $3 \quad$ November, 1970}

John D. Arrison and Michael Rich, On nearly commutative degree one algebras . . . 533

Bruce Alan Barnes, Algebras with minimal left ideals which are Hilbert spaces . . . . 537

Robert F. Brown, An elementary proof of the uniqueness of the fixed point index . . . 549

Ronn L. Carpenter, Principal ideals in F-algebras .................... 559

Chen Chung Chang and Yiannis (John) Nicolas Moschovakis, The Suslin-Kleene

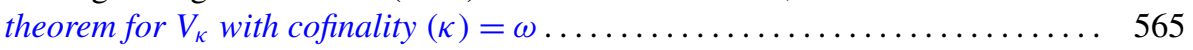

Theodore Seio Chihara, The derived set of the spectrum of a distribution

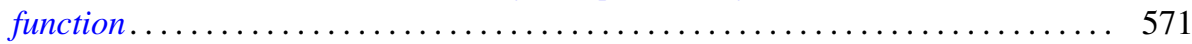

Tae Geun Cho, On the Choquet boundary for a nonclosed subspace of $C(S) \ldots \ldots \quad 575$

Richard Brian Darst, The Lebesgue decomposition, Radon-Nikodym derivative,

conditional expectation, and martingale convergence for lattices of sets .......

David E. Fields, Dimension theory in power series rings . . . . . . . . . . . .

Michael Lawrence Fredman, Congruence formulas obtained by counting

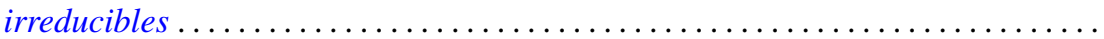

John Eric Gilbert, On the ideal structure of some algebras of analytic functions.....

G. Goss and Giovanni Viglino, Some topological properties weaker than

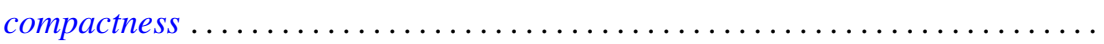

581

601

625

George Grätzer and J. Sichler, On the endomorphism semigroup (and category) of

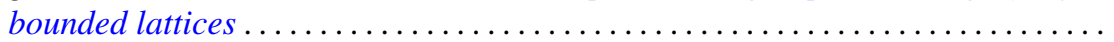

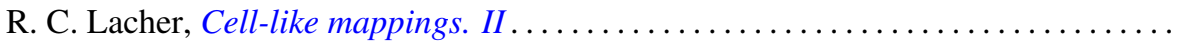

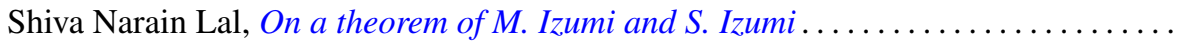

661

Howard Barrow Lambert, Differential mappings on a vector space ...............

Richard G. Levin and Takayuki Tamura, Notes on commutative power joined

semigroups.

Robert Edward Lewand and Kevin Mor McCrimmon, Macdonald's theorem for quadratic Jordan algebras.

J. A. Marti, On some types of completeness in topological vector spaces ....

Walter J. Meyer, Characterization of the Steiner point

717

Saad H. Mohamed, Rings whose homomorphic images are $q$-rings ...

727

Thomas V. O'Brien and William Lawrence Reddy, Each compact orientable surface

of positive genus admits an expansive homeomorphism ...

737

Robert James Plemmons and M. T. West, On the semigroup of binary relations...

743

Calvin R. Putnam, Unbounded inverses of hyponormal operator . .

755

William T. Reid, Some remarks on special disconjugacy criteria for differential

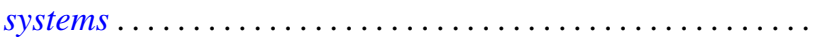

C. Ambrose Rogers, The convex generation of convex Borel sets in euclidean

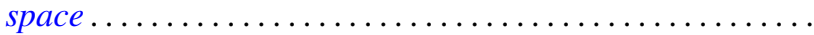

S. Saran, A general theorem for bilinear generating functions .

S. W. Smith, Cone relationships of biorthogonal systems ......

Wolmer Vasconcelos, On commutative endomorphism rings ....

795

Vernon Emil Zander, Products of finitely additive set functions from Orlicz

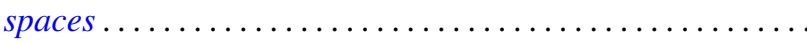

G. Sankaranarayanan and C. Suyambulingom, Correction to: "Some renewal

theorems concerning a sequence of correlated random variables" .

Joseph Zaks, Correction to: "Trivially extending decompositions of $E^{n}$ ”....... 805

Dong Hoon Lee, Correction to: "The adjoint group of Lie groups" ............ 805

James Edward Ward, Correction to: "Two-groups and Jordan algebras". 